\title{
MR Imaging Features of Acute Mastoiditis and Their Clinical Relevance
}

\author{
R. Saat, A.H. Laulajainen-Hongisto, G. Mahmood, L.J. Lempinen, A.A. Aarnisalo, A.T. Markkola, and J.P. Jero
}

\begin{abstract}
BACKGROUND AND PURPOSE: MR imaging is often used for detecting intracranial complications of acute mastoiditis, whereas the intratemporal appearance of mastoiditis has been overlooked. The aim of this study was to assess the imaging features caused by acute mastoiditis in MR imaging and their clinical relevance.
\end{abstract}

MATERIALS AND METHODS: Medical records and MR imaging findings of 31 patients with acute mastoiditis ( 21 adults, 10 children) were analyzed retrospectively. The degree of opacification in the temporal bone, signal and enhancement characteristics, bone destruction, and the presence of complications were correlated with clinical history and outcome data, with pediatric and adult patients compared.

RESULTS: Most patients had $\geq 50 \%$ of the tympanic cavity and $100 \%$ of the mastoid antrum and air cells opacified. Compared with CSF, they also showed intramastoid signal changes in T1 spin-echo, T2 TSE, CISS, and DWI sequences; and intramastoid, outer periosteal, and perimastoid dural enhancement. The most common complications in MR imaging were intratemporal abscess (23\%), subperiosteal abscess (19\%), and labyrinth involvement (16\%). Children had a significantly higher prevalence of total opacification of the tympanic cavity ( $80 \%$ versus $19 \%$ ) and mastoid air cells ( $90 \%$ versus $21 \%$ ), intense intramastoid enhancement (90\% versus $33 \%$ ), outer cortical bone destruction (70\% versus $10 \%$ ), subperiosteal abscess (50\% versus $5 \%$ ), and perimastoid meningeal enhancement ( $80 \%$ versus $33 \%)$.

CONCLUSIONS: Acute mastoiditis causes several intra- and extratemporal changes on MR imaging. Total opacification of the tympanic cavity and the mastoid, intense intramastoid enhancement, perimastoid dural enhancement, bone erosion, and extracranial complications are more frequent in children.

ABBREVIATIONS: $\mathrm{AM}=$ acute mastoiditis; $\mathrm{SI}=$ signal intensity; $\mathrm{SNHL}=$ sensorineural hearing loss

A cute mastoiditis (AM) is a complication of otitis media in which infection in the middle ear cleft involves the mucoperiosteum and bony septa of the mastoid air cells. It can be divided into coalescent and noncoalescent mastoiditis. In coalescent AM, infection causes osteolysis of the bony septa or cortical bone, which can further lead to intra- and extracranial complications.

AM diagnosis is usually based on clinical findings, with imaging useful for detecting complications or ruling out other disease

Received April 24, 2014; accepted after revision August 7.

From the Department of Radiology, HUS Medical Imaging Center (R.S., G.M., A.T.M.), and Department of Otorhinolaryngology and Head and Neck Surgery (A.H.L.-H., L.J.L., A.A.A., J.P.J.), Helsinki University Central Hospital and University of Helsinki, Helsinki, Finland; and Department of Radiology (R.S.), East Tallinn Central Hospital, Tallinn, Estonia.

The study was supported by the Helsinki University Central Hospital Research Funds.

Please address correspondence to Riste Saat, MD, Department of Radiology, HUS Medical Imaging Center, POB 340, Haartmaninkatu 4, HUS 00029 Helsinki, Finland; e-mail: riste.saat@hus.fi

http://dx.doi.org/10.3174/ajnr.A4120 entities mimicking AM. ${ }^{1,2}$ Treatment is mainly conservative, with mastoidectomy reserved for those with complications or no response to adequate antimicrobial treatment. ${ }^{3,4}$ However, generally accepted guidelines for the treatment of AM are lacking, and treatment algorithms vary by institution.

CT is usually the initial technique of choice for imaging patients with AM. Intravenous contrast agent is advisable for better evaluation of perimastoid soft tissues and because some intracranial complications like venous sinus thrombosis are detectable only from contrast-enhanced images. MR imaging is mainly reserved for detection or detailed evaluation of intracranial complications or both.

While the usefulness of MR imaging in diagnosing intracranial AM spread has been demonstrated many times over, ${ }^{1,5-9}$ intratemporal findings of AM on MR imaging tend to be overlooked and information on their clinical relevance is scarce. Problems exist with overdiagnosing "mastoiditis" on MR imaging if it is based on intramastoid fluid signal alone. ${ }^{10,11}$ Because MR imaging use in clinical practice is increasing, precise information on 
Table 1: Distribution of intramastoid signal intensity and enhancement

\begin{tabular}{|c|c|c|c|c|}
\hline \multirow[b]{2}{*}{$\mathrm{SI}$ in T2 FSE and CISS } & \multirow[t]{2}{*}{ No. } & \multicolumn{3}{|c|}{ No. of Patients per Category (Valid \%) } \\
\hline & & Isointense to CSF & Hypointense to CSF, hyperintense to WM & Iso- or hypointense to WM \\
\hline CISS & 25 & $1(4)$ & $14(56)$ & $10(40)$ \\
\hline T2 FSE & 31 & $3(10)$ & $24(77)$ & $4(13)$ \\
\hline SI in TI SE & & Isointense to CSF & Hyperintense to CSF, not to WM & Hyperintense to WM \\
\hline TI SE & 31 & $0(0)$ & $22(71)$ & $9(29)$ \\
\hline \multirow{2}{*}{ SI in DWI $(b=1000)$} & & Hypointense to WM & Isointense to WM & Hyperintense to WM \\
\hline & 27 & $2(7)$ & $9(33)$ & $16(59)$ \\
\hline \multirow[t]{2}{*}{$\mathrm{SI}$ in $\mathrm{ADC}$} & & Not lowered & Lowered & \\
\hline & 26 & $10(38)$ & $16(62)$ & - \\
\hline \multirow[t]{2}{*}{ Enhancement } & & None & Faint, thin & Intense, thick \\
\hline & 31 & $3(10)$ & $12(39)$ & $16(52)$ \\
\hline
\end{tabular}

Note:-No. indicates the number of patients with a specific sequence available.

the spectrum of MR imaging features of AM is essential. Our aim was to describe MR imaging findings resulting from AM and to clarify their clinical relevance.

\section{MATERIALS AND METHODS \\ Patients}

All 153 patients with a discharge diagnosis of AM (International Classification of Diseases-10 code H70.0) in the Ear, Nose, and Throat Department of our institution (a tertiary referral center providing health care for approximately 1.5 million people) during a 10-year period (2003-2012) were retrospectively identified from the hospital data base. Based on recent reports, ${ }^{12,13}$ the diagnostic criteria for AM in our institution were the following: either intraoperatively proved purulent discharge or acute infection in the mastoid process, or findings of acute otitis media and at least 2 of these 6 symptoms: protrusion of the pinna, retroauricular redness, retroauricular swelling, retroauricular pain, retroauricular fluctuation, or abscess in the ear canal, with no other medical condition explaining these findings. Those with MR imaging of the temporal bones available $(n=34)$ were selected for this study. We excluded 3 patients: 1 with recurrent disease after previous mastoidectomy, 1 with secondary inflammation due to an underlying tumor, and 1 in whom an intraoperative biopsy revealed middle ear sarcoidosis. The final analysis covered 31 patients. The study protocol was approved by the institutional ethics committee.

\section{Clinical Data}

Clinical data were collected from electronic patient records and consisted of the following variables: age and sex, side of the AM, duration of symptoms, duration of intravenous antibiotic treatment, presence or absence of retroauricular signs of infection (redness, swelling, pain, fluctuation, protrusion of the pinna), sensorineural hearing loss (SNHL), decision for operative treatment, mastoidectomy, and duration of hospitalization.

\section{MR Imaging Protocol}

MR imaging examinations were performed on a 1.5T unit (Magnetom Avanto; Siemens, Erlangen, Germany) with a 12-channel head and neck coil in 30 patients and on a 3T unit (Achieva; Philips Healthcare, Best, Netherlands) with an 8-channel head coil in 1 patient. The standard MR imaging protocol for mastoiditis consisted of axial and coronal T2 FSE and axial T1 spin-echo images, axial EPI DWI (b factors of 0 and $1000 \mathrm{~s} / \mathrm{mm}^{2}$ ) and an
ADC map with 3-mm section thickness, high-resolution T2weighted CISS images with $0.7-\mathrm{mm}$ section thickness, and T1 MPRAGE images after intravenous administration of 0.1 $\mathrm{mmol} / \mathrm{kg}$ of body weight of gadoterate meglumine (Dotarem; Guerbet, Aulnay-sous-Bois, France), obtained in the sagittal plane and reconstructed as $1-\mathrm{mm}$ sections in axial and coronal planes.

\section{Image Interpretation}

The MR images were independently analyzed for their consensus diagnosis by 2 board-certified radiologists (R.S. and G.M. with 6 and 3 years of experience in reading temporal bone MR images and each holding a Certificate of Added Qualification in, respectively, head and neck radiology and neuroradiology). For every patient, only 1 ear was evaluated. In the 1 case with bilateral mastoiditis, only the first-involved ear was included.

Opacification degree in the tympanic cavity, mastoid antrum, and mastoid air cells; signal intensity in T1 spin-echo, T2 FSE, CISS, and DWI $(b=1000)$; and intramastoid enhancement were recorded and scored into 3-4 categories of increasing severity by the principles shown in Table 1 and Fig 1. The following imaging findings were reported as being either present or absent: drop in signal intensity on the ADC map, blockage of the aditus ad antrum, bone destruction, signs of intratemporal abscess, signs of inflammatory labyrinth involvement, enhancement of the outer periosteum, perimastoid dural enhancement, epidural abscess, subperiosteal abscess, subdural empyema, generalized pachymeningitis, leptomeningeal enhancement, soft-tissue abscess, or sinus thrombosis.

Mastoid pneumatization is variable among patients and its contents inhomogenous, making objective signal intensity (SI) measurements complicated. Therefore, the intramastoid MR imaging SI was evaluated subjectively from the most abnormal regions in comparison with the SI of cerebellar WM in the same image and with the CSF in the location with no pulsation artifacts. It was scored according to the highest on T1WI and DWI $(b=1000)$ or the lowest on T2WI detectable SI that involved a substantial part of the mastoid process. Additionally, ADC values were subjectively estimated as being either lowered or not lowered.

Destruction of bony structures was estimated from T2 FSE images as loss of morphologic integrity of bony structures or clear signal transformation inside the otherwise signal-voided cortical bone. This was evaluated at 3 subsites: the intercellular 

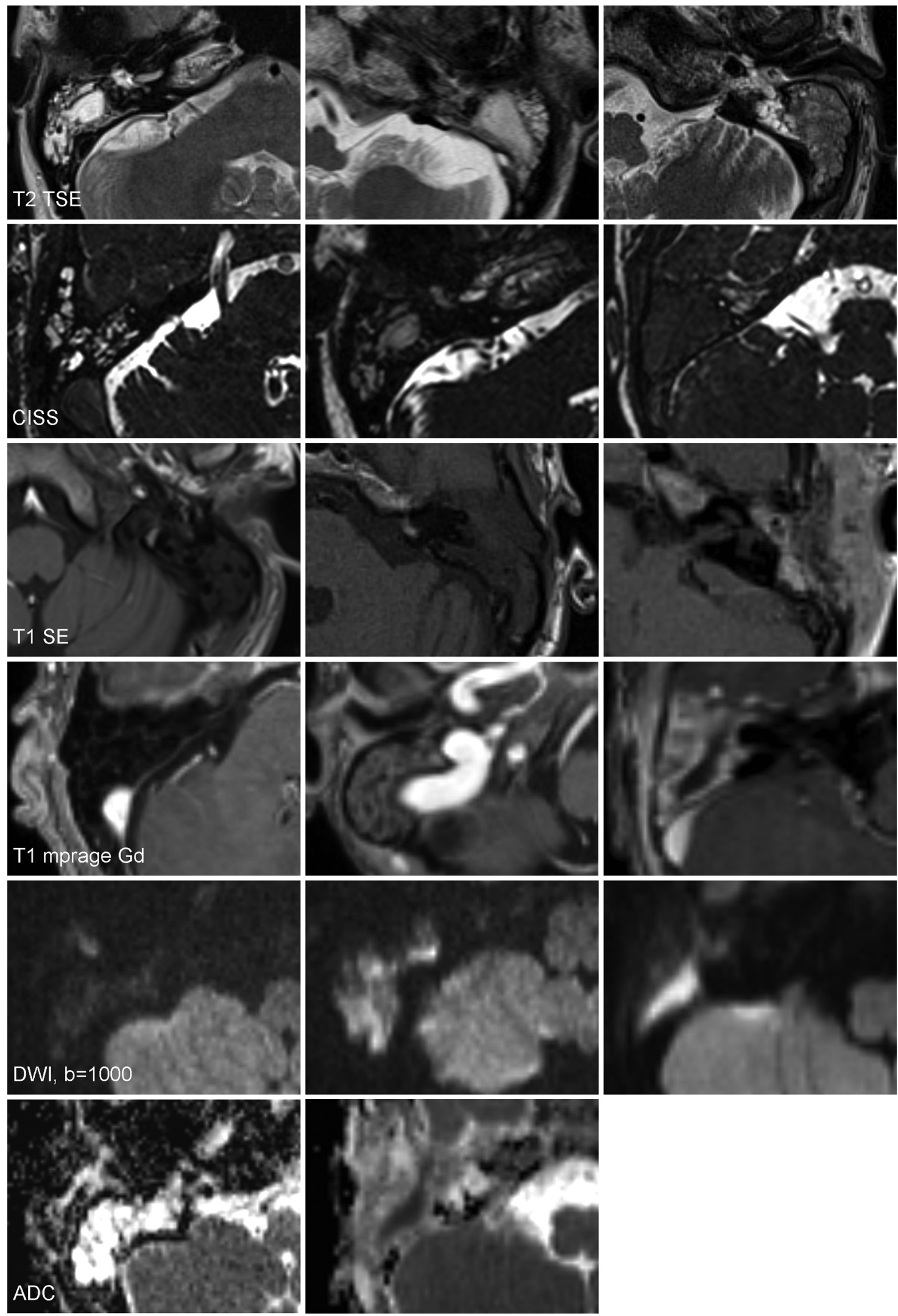

FIG 1. Image examples of each scoring category according to signal intensities. Categories are displayed in columns from left to right in increasing severity.

bony septa of the mastoid, inner cortical bone toward the intracranial space, and outer cortical bone toward the extracranial soft tissues.
Blockage of the aditus ad antrum was defined as filling of the aditus lumen by enhanced tissue. Signs of inflammatory labyrinth involvement were either diffuse intralabyrinthine en- 
hancement or perilymph signal drop in CISS. Intratemporal abscess was defined as a nonanatomic cavity inside the temporal bone with an enhanced wall and marked diffusion restriction inside it. Criteria for generalized pachymeningitis (in contrast to perimastoid dural enhancement) were extensive thickening and enhancement of the dura that extended past the borders of the temporal bone.

\section{Statistical Analysis}

Due to the relatively small number of patients, the original MR imaging scoring groups were dichotomized by summation of the original scoring groups into groups of comparable sizes before statistical analysis. Associations between dichotomized MR imaging findings and background or outcome parameters were determined with the Fisher exact test for categoric data and the Mann-Whitney $U$ test for numeric data. Clinical aspects and imaging findings between pediatric and adult patient groups were compared with the Fisher exact test. Statistical analysis was conducted by a biostatistician with NCSS 8 software (NCSS, Kaysville, Utah). A $P$ value of $<.05$ was considered statistically significant.

\section{RESULTS}

\section{Patients and Clinical Data}

Thirty-one patients were analyzed (11 male and 20 female); mean age, 33.4 years (range, 3-81 years). Age distribution showed 2 peaks between 10 and 20 and between 40 and 50 years. Pediatric patients (16 years of age or younger) numbered 10 .

The average duration of symptoms before MR imaging was 12.9 days (range, 0-90 days). Classic retroauricular signs of mastoid infection were present in 18 patients (58\%); and SNHL in 15 (48\%). Intravenous antibiotics had been initiated for at least 24 hours before MR imaging in 18 patients (58\%); and the mean duration of this treatment was 2.8 days (range, 0-22 days).

Operative treatment was chosen for 20 patients $(65 \%)$, and mastoidectomy was performed for 19 (61\%) because of parent refusal in 1 patient.

The average length of hospitalization was 6.7 days (range, 1-26 days). Six patients had recurrent symptoms within the 3-month follow-up.

\section{MR Imaging Findings}

Opacification of the Middle Ear and Mastoid. Most patients had at least a $50 \%$ opacification in the tympanic cavity and total opacification of the mastoid antrum and air cells (Fig 2). A significant correlation appeared between $\geq 50 \%$ opacification in the tympanic cavity and longer intravenous antibiotic treatment (mean, 5.0 versus 2.0 days; $P=.031$ ).

Intramastoid Signal Intensities. Findings regarding intramastoid signal intensities are demonstrated in Table 1. On T2 FSE, among 31 patients, the SI was hypointense to CSF in 28 (90\%) and iso- or hypointense to WM of the brain in 4 (13\%). On CISS, among 25 patients, SI was hypointense to CSF in 24 (96\%) and iso- or hypointense to WM in 10 (40\%). On unenhanced T1 spin-echo, SI was hyperintense to CSF in all 31 patients and hyperintense to

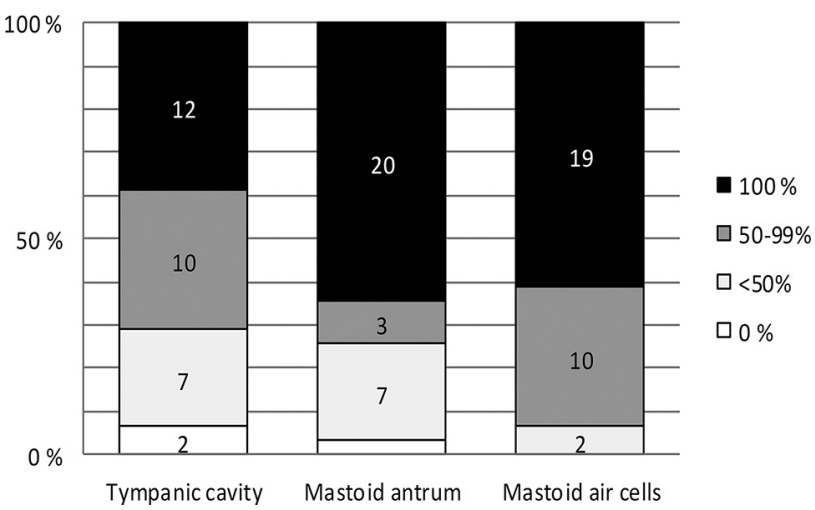

FIG 2. Obliteration degree in different temporal bone subregions $(n=31)$.

WM in $9(29 \%)$. On DWI $(b=1000)$, among 27 patients, SI was iso-or hyperintense to WM in 25 (93\%) and hyperintense to WM in $16(59 \%)$. Lowered SI in the ADC was detectable in 16 of 26 patients (62\%). Hyperintense-to-WM SI in DWI was associated with a shorter duration of intravenous antibiotic treatment (mean, 1.9 versus 5.0 days; $P=.029$ ).

Enhancement Characteristics. Intramastoid enhancement was detectable in 28 patients (90\%) and was thick and intense in 16 (52\%) (Fig 3). Intense enhancement was associated with younger age (mean, 24.6 versus 42.7 years; $P=.019$ ). Enhancement of the outer periosteum occurred in 21 patients $(68 \%)$; and perimastoid dural enhancement, in 15 (48\%). Outer periosteal enhancement correlated with shorter duration of symptoms (7.1 versus 25.1 days, $P=.009)$. Obliteration of the aditus ad antrum by enhanced tissue was detected in 11 patients (36\%).

Bone Destruction. Destruction of the intramastoid bony septa was suspected in 11 (35\%); of inner cortical bone, in 4 (13\%); and of outer cortical bone, in 9 (29\%) patients. Destruction of outer cortical bone was associated with younger age (mean, 34.0 versus 48.7 years; $P=.004)$, shorter duration of symptoms before MR imaging (mean, 11.0 versus 24.5 days; $P=.031$ ), and the presence of retroauricular signs of infection $(P=.045)$.

Complications. Intratemporal and extracranial complications predominated over intracranial complications (Table 2). Intratemporal abscess formation was suspected in 7 patients (23\%). Subperiosteal abscesses were detectable in $6(19 \%)$ and were correlated with younger age (mean, 6.0 versus 25.0 years; $P=.010$ ) and with retroauricular signs of infection $(P=.028)$. Labyrinth involvement was detectable in 5 patients $(16 \%)$. The prevalence of other complications was low in our cohort: 2 (7\%) with epidural abscess, generalized pachymeningitis, leptomeningitis, or soft-tissue abscess; 1 (3\%) with sinus thrombosis; and none with subdural empyema.

Outcome. Opacification of the tympanic cavity of $100 \%$ was associated positively with the decision for operative treatment $(P=.020)$.

All patients with labyrinth involvement on MR imaging had SNHL $(P=.043)$. Additionally, SNHL was associated with obliteration of the aditus ad antrum by enhanced tissue $(P=.023)$ and outer cortical bone destruction $(P=.015)$. 

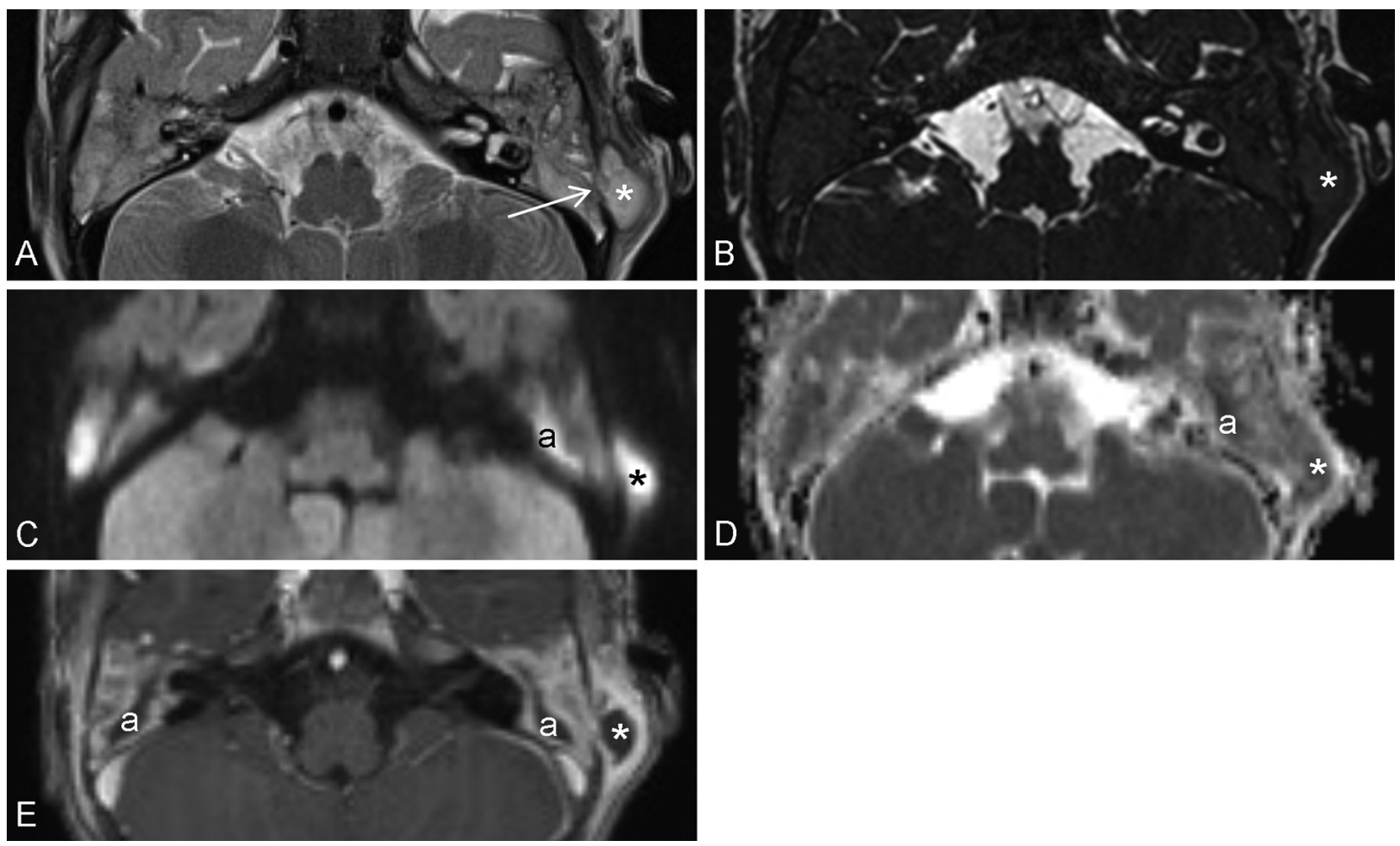

FIG 3. MR images of bilateral AM with duration of symptoms of 12 days on the left and fewer than 6 days ( $3-6$ days) on the right side. T2 FSE image $(A)$ shows total obliteration of middle ear and mastoid air spaces. SI is comparable with that of brain parenchyma. Especially on the right side, delineation of intramastoid bony septa is no longer detectable. On the left, outer cortical bone is destroyed (arrow) with subperiosteal abscess formation (asterisk). Intramastoid signal decrease, compared with CSF, becomes even more evident in CISS (B). In postgadolinium TI MPRAGE (E), intense, thick enhancement surrounds the fluid-filled mastoid antra (a) and fills the peripheral mastoid cells. On the left, intense soft-tissue enhancement around the subperiosteal abscess and, on the right, periosteal enhancement surrounding the mastoid are visible. DWI $b=1000(C)$ and ADC (D) show diffusion restriction in the whole mastoid region bilaterally with foci of markedly elevated SI inside both antra (a) and the left subperiosteal abscess (asterisk).

Table 2: Prevalence of AM complications detected on MRI $(N=31)$

\begin{tabular}{llll}
\hline & \multicolumn{3}{c}{ No. (\%) } \\
\cline { 2 - 4 } \multicolumn{1}{c}{ Complication } & All & Children & Adults \\
\hline Septal destruction & $11(35)$ & $5(45)$ & $6(30)$ \\
Inner cortical bone destruction & $4(13)$ & $2(18)$ & $2(10)$ \\
Outer cortical bone destruction & $9(29)$ & $7(64)^{\mathrm{a}}$ & $2(10)^{\mathrm{a}}$ \\
Intratemporal abscess & $7(23)$ & $3(27)$ & $4(20)$ \\
Subperiosteal abscess & $6(19)$ & $5(45)^{\mathrm{a}}$ & $1(5)^{\mathrm{a}}$ \\
Labyrinth involvement & $5(16)$ & $0(0)$ & $5(25)$ \\
Epidural abscess & $2(6)$ & $2(18)$ & $0(0)$ \\
Soft-tissue abscess & $2(6)$ & $1(9)$ & $1(5)$ \\
Generalized pachymeningitis & $2(6)$ & $1(9)$ & $1(5)$ \\
Leptomeningitis & $2(6)$ & $1(9)$ & $1(5)$ \\
Sinus thrombosis & $1(3)$ & $1(9)$ & $0(0)$ \\
Subdural empyema & $0(0)$ & $0(0)$ & $0(0)$ \\
\hline
\end{tabular}

${ }^{a}$ Significant differences between adult and pediatric subgroups $(P<.05)$.

Pediatric versus Adult Patients. In pediatric patients, a significantly higher prevalence of total opacification occurred in the tympanic cavity ( $80 \%$ versus $19 \%, P=.002)$ and mastoid air cells (90\% versus $21 \%, P=.046)$. Children more frequently showed intense intramastoid enhancement (90\% versus 33\% $P=.006$ ), enhancement of the perimastoid dura (80\% versus 33\%, $P=$ $.023)$, possible outer cortical bone destruction (70\% versus $10 \%$, $P=.001$ ), and subperiosteal abscess (50\% versus 5\%, $P=.007$ ). Accordingly, among children, the prevalence of retroauricular signs of infection was also higher ( $90 \%$ versus $43 \%, P=.020)$.

\section{DISCUSSION}

Imaging plays an important role in AM diagnostics, especially in complicated cases. The imaging technique of choice usually is CT for its sensitivity in detecting opacification and bone destruction. Its capability to differentiate among causes of opacification is poor.

In comparison with $\mathrm{CT}$, MR imaging performs better in differentiating among soft tissues and in showing juxtaosseous contrast medium uptake, due to the natural MR signal void in bone. In the context of AM, evidence indicates the superiority of MR imaging over CT in the detection of labyrinth involvement and intracranial infection. ${ }^{1,6,14}$ Little focus has, however, been on intratemporal MR imaging findings, with most reports only of intramastoid high signal intensity on T2WI, reflecting fluid retention-a finding evidently nonspecific and leading to mastoiditis overdiagnosis. ${ }^{10,11}$

During mastoiditis, variable signal intensities of retained fluid and intratemporal enhancement can appear, explained by desiccation of fluids and overgrowth of granulation tissue, especially under chronic conditions. ${ }^{8}$ According to Platzek et al ${ }^{15}$ (2014) a sensitivity of $100 \%$ and specificity of $66 \%$ in diagnosing AM are possible, with $\geq 2$ of these intramastoid findings: fluid accumulation, enhancement, or diffusion restriction. In most of our patients with $\mathrm{AM},>50 \%$ opacification of air spaces occurred in all temporal bone subregions (Fig 2). Total opacification of the tym- 
panic cavity was the only imaging finding significantly associated with treatment options. Although opacification degree in the tympanic cavity usually was lower than that in the distal parts of the temporal bone, when $100 \%$, it indicated a decision to perform surgery. All our patients had, before the MR imaging, either existing tympanic membrane perforation or myringotomy or a tympanostomy tube in place. In patients with an intact tympanic membrane, opacification of the tympanic cavity may have a different prognostic impact.

Trends toward predicting operative treatment were also detectable in regard to total opacification of mastoid air cells $(P=$ .056) and thick and intense intramastoid enhancement $(P=$ .066). In larger cohorts, these may still prove valuable markers of severe disease.

In most patients ( $\geq 90 \%$ ), intramastoid signal intensity on $\mathrm{T} 2$ TSE and even more on CISS was lower than that of CSF and even reached the values of the white matter SI (Table 1), most likely due to the increased protein content of the obliterating material. On T1WI, SI of the intramastoid substance, in comparison with CSF, was increased in all patients.

In some patients, marked signal changes and intense intramastoid enhancement were detected early in AM, even on the second symptomatic day, and therefore cannot be related to chronic conditions only. ${ }^{8}$

DWI was included in our protocol to detect purulent secretions and possible intratemporal abscesses. ${ }^{16-20}$ On DWI, most patients (93\%) showed variable degrees of signal increase in their mastoid effusions (Table 1). Nearly two-thirds (59\%) had intramastoid signal intensity higher than that in their brain parenchyma on DWI and low signal on ADC, confirming the true diffusion restriction.

In contrast to cholesteatoma, diffusion restriction in AM is usually more diffuse. ${ }^{21}$ In cases of cholesteatoma underlying mastoiditis or in mastoiditis complicated by intratemporal abscess, difficulties may arise, calling for either surgical exploration or follow-up imaging. Differentiation among cholesteatoma, infected cholesteatoma, and intratemporal abscess may be possible, based on their ADC values, though large-study evidence is still lacking. ${ }^{22}$

Imaging findings associated with either a clinically rapid course and shorter duration of symptoms or shorter duration of IV antibiotic treatment before MR imaging were outer periosteal enhancement, destruction of outer cortical bone, and hyperintense-to-WM SI on DWI. Outer cortical destruction and subperiosteal abscesses were associated with clinical signs of retroauricular infection.

Compared with adults, children, especially at a younger age (younger than 2 years) generally tend to develop so-called classic AM- usually of short duration and rapid course, with distinct clinical symptoms and signs. ${ }^{12,13}$ Our pediatric patients more often showed total opacification of the tympanic cavity and mastoid, strong intramastoid enhancement, outer cortical bone destruction, and subperiosteal abscesses. Intracranial complications were no more numerous among children when compared with adults, but these were very rare in each subgroup.

Our limitations are the small size and inhomogeneity of the patient cohort. For patients with AM, MR imaging was performed rarely, usually for severe disease or unsatisfactory treatment response. Our imaging series thus does not reflect the average AM population. It includes both hyperacute cases and patients with a longer history and antibiotic treatment for variable durations. Drawing firm conclusions regarding the prognostic value of these MR imaging findings is thus difficult.

Traditionally in our institution, imaging was performed to confirm suspicion of AM complications necessitating surgery. In other circumstances, treatment decisions were based solely on clinical evidence of progressive disease, failure to respond to IV antibiotics within 48 hours, or underlying cholesteatoma. ${ }^{23}$

In clinical practice, contrast-enhanced CT is still the preferable, first-line imaging technique due to better availability in urgent situations. Otologists are more familiar with CT images as their preoperative map. In addition to detecting intracranial complications, MR imaging could be recommended for pediatric patients due to its lack of ionizing radiation. In young children, however, CT may be preferred over MR imaging when anesthesia is inadvisable. MR imaging provides an alternative diagnostic tool for patients with contraindications for contrast-enhanced CT and could benefit decision-making concerning surgery in conservatively treated patients with insufficient clinical response.

Thus far, radiologic markers for aggressive AM have been either bone destruction in CT or intra- and extracranial complications. Now MR imaging provides additional imaging markers reflecting soft-tissue reaction to infection: major intramastoid signal changes; diffusion restriction; or intramastoid, periosteal, or dural enhancement. These may serve in the assessment of AM severity. Their accuracy in detecting clinically relevant AM and their true prognostic value remain to be clarified by larger studies.

\section{CONCLUSIONS}

Acute mastoiditis causes several intra- and perimastoid changes visible on MR imaging, with $>50 \%$ opacification of air spaces, non-CSF-like signal intensity of intramastoid contents, and intramastoid and outer periosteal enhancement detectable in most patients. In children, total opacification of the tympanic cavity and mastoid, intense intramastoid enhancement, perimastoid dural enhancement, bone erosion, and extracranial complications are more frequent.

\section{ACKNOWLEDGMENTS}

The authors thank Timo Pessi, MSc, for his assistance with statistics and Carolyn Brimley Norris, $\mathrm{PhD}$, for her linguistic expertise.

Disclosures: Anu H. Laulajainen-Hongisto—RELATED: Grant: Helsinki University Central Hospital (research funds); Support for Travel to Meetings for the Study or Other Purposes: Finnish Society of Ear Surgery, Comments: Politzer Society meeting. Jussi P. Jero-RELATED: Grant: Helsinki University Hospital.* * Money paid to the institution.

\section{REFERENCES}

1. Minks DP, Porte M, Jenkins N. Acute mastoiditis: the role of radiology. Clin Radiol 2013;68:397-405

2. Psarommatis IM, Voudouris C, Douros K, et al. Algorithmic management of pediatric acute mastoiditis. Int J Pediatr Otorhinolaryngol 2012;76:791-96

3. Bakhos D, Trijolet J, Morniere S, et al. Conservative management of acute mastoiditis in children. Arch Otolaryngol Head Surg 2011;137:346-50 
4. Chesney J, Black A, Choo D. What is the best practice for acute mastoiditis in children? Laryngoscope 2014;124:1057-58

5. Luntz M, Bartal K, Brodsky A, et al. Acute mastoiditis: the role of imaging for identifying intracranial complications. Laryngoscope 2012;122:2813-17

6. Dobben GD, Raofi B, Mafee MF, et al. Otogenic intracranial inflammations: role of magnetic resonance imaging. Top Magn Reson Imaging 2000;11:76-86

7. Hughes DC, Raghavan A, Mordekar SR, et al. Role of imaging in the diagnosis of acute bacterial meningitis and its complications. Postgrad Med J 2010;86:478-85

8. Maroldi R, Farina D, Palvarini L, et al. Computed tomography and magnetic resonance imaging of pathologic conditions of the middle ear. Eur J Radiol 2001;40:78-93

9. Vazquez E, Castellote A, Piqueras J, et al. Imaging of complications of acute mastoiditis in children. Radiographics 2003;23: $359-72$

10. Polat S, Aksoy E, Serin GM, et al. Incidental diagnosis of mastoiditis on MRI. Eur Arch Otorhinolaryngol 2011;268:1135-38

11. Meredith J, Boyev K. Mastoiditis on MRI: fact or artifact? Ear Nose Throat J 2008;87:514-18

12. Groth A, Enoksson F, Hultcrantz M, et al. Acute mastoiditis in children aged 0-16 years: a national study of 678 cases in Sweden comparing different age groups. Int J Pediatr Otorhinolaryngol 2012;76:1494-500

13. Stalfors J, Enoksson F, Hermansson A, et al. National assessment of validity of coding of acute mastoiditis: a standardised reassessment of 1966 records. Clin Otolaryngol 2013;38:130-35

14. Kuczkowski J, Dubaniewicz-Wybieralska M, Przewozny T, et al.
Otitic hydrocephalus associated with lateral sinus thrombosis and acute mastoiditis in children. Int $J$ Pediatr Otorhinolaryngol 2006;70:1817-23

15. Platzek I, Kitzler H, Gudziol V, et al. Magnetic resonance imaging in acute mastoiditis. Acta Radiol Short Rep 2014;3:2047981614523415

16. Schaefer PW. Applications of DWI in clinical neurology. J Neurol Sci 2001;186(suppl 1):S25-35

17. Lai PH, Ho JT, Chen WL, et al. Brain abscess and necrotic brain tumor: discrimination with proton MR spectroscopy and diffusion-weighted imaging. AJNR Am J Neuroradiol 2002;23:1369-77

18. Mukherji SK, Chenevert TL, Castillo M. Diffusion-weighted magnetic resonance imaging. J Neuroophthalmol 2002;22:118-22

19. Stadnik TW, Chaskis C, Michotte A, et al. Diffusion-weighted MR imaging of intracerebral masses: comparison with conventional MR imaging and histologic findings. AJNR Am J Neuroradiol 2001;22:969-76

20. Unal O, Koparan HI, Avcu S, et al. The diagnostic value of diffusionweighted magnetic resonance imaging in soft tissue abscesses. Eur J Radiol 2011;77:490-94

21. Vercruysse JP, De Foer B, Pouillon M, et al. The value of diffusionweighted MR imaging in the diagnosis of primary acquired and residual cholesteatoma: a surgical verified study of 100 patients. Eur Radiol 2006;16:1461-67

22. Thiriat S, Riehm S, Kremer S, et al. Apparent diffusion coefficient values of middle ear cholesteatoma differ from abscess and cholesteatoma admixed infection. AJNR Am J Neuroradiol 2009;30:1123-26

23. Leskinen K, Jero J. Acute complications of otitis media in adults. Clin Otolaryngol 2005;30:511-16 